\title{
Chronic Consequences of Uncomplicated Yersinia Enterocolitica Infection: A Retrospective Study (2004) of Military Recruits Previously (1987) Screened for Antibodies to Yersinia Enterocolitica
} 0:3

\author{
Arve Sæbø $^{1}$, Karin Nygard ${ }^{2}$, Georg Kapperud ${ }^{2,3}$, Jørgen Lassen ${ }^{2}$ \\ ${ }^{1}$ Department of Surgery, Molde Hospital, Molde, Norway; ${ }^{2}$ Division of Infectious Disease Control, Norwegian Institute of Public \\ Health, Oslo, Norway; ${ }^{3}$ Norwegian School of Veterinary Science, Oslo, Norway. \\ Email: asaebo@online.no
}

Received February $8^{\text {th }}, 2011$; revised March 10 ${ }^{\text {th }}, 2011$; accepted March $28^{\text {th }}, 2011$.

\begin{abstract}
In 1987, a serological survey among 755 male Norwegian military recruitsshowed that 68 (9.0\%) had IgG (7.4\%) and/or IgA (4.5\%) antibodiesto Y. enterocolitica O:3, consistent with previous infection. In 2004, 433 of the former recruits (mean age 37.5 years, range 35 - 42 years) answered a questionnaire, in order to investigate development of possible chronic disorders and complaints related to uncomplicated Y. enterocolitica infection. The questionnaire covered personal and demographic data, behavioral risk factors, and possible chronic complaints related to yersiniosis. Among the 433 responders, 51 (11.8\%) were seropositive for IgG or IgA in the 1987 survey, 380 were seronegative, and 2 had ambiguous results. No difference was observed between the seropositive and seronegative groups regarding education, annual income, general behavioral risk factors like smoking, alcohol consumption, physical activity, and dietary parameters; or diagnosed chronic illness. However, the seropositives were significantly more likely than the seronegatives to report recurrent diarrhea, painful or swollen joints, and muscle pain. Also, presence of all three complaints was more common among seropositives. Conclusion: The present study shows that uncomplicated Y. enterocolitica O:3 infection is a risk factor regarding future development of chronic complaints such as diarrhea, and joint and muscle complaint.
\end{abstract}

Keywords: Chronic Complaint (Diarrhea, Painful/Swollen Joints, Muscle Pain)

\section{Introduction}

Yersinia enterocolitica can cause a range of acute clinical entities including gastro-intestinal disease, hepatitis, pancreatitis, nephritis, arthritis, myalgia, erythema nodosum etc., and multi-organ involvement [1-5]. The Y. enterocoliticainfection may also provoke chronic rheumatic disorders, most notably in patients with the histo compatibility antigen HLA-B27 [6,7]. Clinical and pathological studies indicate that also chronic conditions as inflammatory bowel disease, chronic hepatitis, nephritis etc., may be induced [5,8-11].

Most knowledge about long-term consequences of $\mathrm{Y}$. enterocolitica infection has been obtained from retrospective or follow-up studies of hospitalized patients [5,
11-14], from case-control studies of culture-confirmed cases [15], and from immuno-histochemical examination of specimens from patients with supposed chronic manifestations of Y. enterocolitica infection [16-18].

However, studies based on patients with diagnosed Y. enterocolitica infection, whether hospitalized or not, are likely to be biased both in terms of the nature and severity of clinical manifestations. More representative data may be obtained from patients identified by screening of the population as whole, or of population subsets.

The objective of the present study was to investigate the possible development of chronic disorders and complaints related to seemingly uncomplicated Y. enteroclitica infection. We therefore, in 2004, conducted a retrospective study onour previous material, constituted by 
recruits enrolled 1987 for compulsory military service at the Norwegian naval training camp [19].

\section{Material and Methods}

\subsection{Material and Observations}

In January 1987, 755 out of 791 (=95\%) previously healthy recruits (all ethnical Norwegians males ,19-26 years old, mean age 20 years), who were enrolled at the Norwegian naval training camp for compulsory military service,participated in a study on prevalence of antibodies to Y. enterocolitica, and association with risk factors and clinical manifestations.

Blood samples were drawn within seven days after enrolment, and submitted to the National Institute of Public Health for examination of $\operatorname{IgG}$, IgM and $\operatorname{IgA}$ antibody response to Y. enterocolitica, using an ELISA (v.i.).

Within two weeks, the recruits answered a standardized questionnaire covering demographic data, specific exposures, and clinical information. Information on peroperative findings in 26 recruits with previous appendectomy was obtained from hospital files.

Antibody activity against $\mathrm{Y}$. enterocolitica was detected in sera from 70 of 755 recruits $(9.3 \%)$ (IgG activity: 56 (7.4\%), IgA activity: 34 (4.5\%), and IgM activity: 28 (3.7\%). Information on IgG antibody activity was used in epidemiological analysis of risk factors. The highest prevalence of IgG antibodies was detected among recruits from Oslo city $(12 / 56,21.4 \%)$. The following risk factors were found to be independently associated with IgG activity in logistic regression analysis: 1) receiving drinking water from a private well (odds ratio $(\mathrm{OR})=$ $3.40, p=0.004)$, being a resident of Oslo city $(\mathrm{OR}=2.99$, $p=0.006)$, and living in eastern Norway $(\mathrm{OR}=2.25, p=$ 0.015 ).

Seropositive recruits were more likely to report previous surgery for suspected appendicitis than seronegatives $(\mathrm{OR}=4.26, p=0.0024)$. Among recruits with previous appendectomy, (severe) mesenteric lymphadenitis as the sole per-operative finding was more common inpatients with IgG activity to $Y$. enterocolitica, than in seroegative patients $(4 / 7>1 / 19, p=0.01)$. Among the four seroposive recruits with mesenteric lymphadenitis, three had undergone surgery 11 years, and one 13 years, prior to enrollment, steatorrhea.

Recurrent diarrhea, steatorrhea joint complaints were not associated with antibody activity [19].

\subsection{Serological Analysis}

The ELISA was performed in flat-bottomed 96-well polystyrene micro-titer plates. Antigen was prepared by hot phenol-water extraction of LPS from a phenol killed reference strain of Y. enterocolitica O:3. The optical den- sity (OD) of the color reaction was read in an automatic photometer (MR 580 Microelisa Auto Reader, Dynatech Instruments, Inc., Santa Monica, California, USA) at a wavelength of $405 \mathrm{~nm}$, with $490 \mathrm{~nm}$ as the reference wavelength (dual mode). The cut-off value for discrimination between positive and negative results was based on data from repeated testing of 30 arbitrarily selected sera from healthy blood donors (mean delta-OD plus two standard deviations). Positive and negative control sera were included on each plate in order to minimize interassay variations, Serum samples were examined for specific IgG, IgM and IgA activity. Net absorbance values (absorbance in the sample minus absorbance in the negative control) of $\geq 0.1$ were recognized as significant regarding IgG and IgA, but not lonely IgM. A net absorbance of $\geq 0.5$ was considered indicative of actual or recent infection. (An extensive procedure description has previously been published [19]).

\subsection{Follow-up Material}

At the follow-up in January 2004, five (all seronegative) of the 755 recruits enrolled in 1987 were dead, 18 had emigrated, and in case of 21 the residence was unknown. A structured questionnaire was mailed to the remaining 711 persons, together with a letter of introduction. The questionnaire covered 1) personal and demographic data (including education and annual income), 2) possible general risk factors for disease development (i.e. smoking, alcohol consumption, physical activity), and 3) dietary parameters (including consumption of meat, fruits, and vegetables; all parameters measured in a quantitative way).

The participants were also questioned whether they had developed particular chronic complaints or disorders since 1987. The clinical entities included were: painful or swollen joints, muscle pains or fibromyalgy, and diarrhea; which might be experienced for shorter or longer periods of time. The disorders were the specific diagnoses of rheumatoid arthritis, ankylosing spondylitis, iridocyclitis, ulcerative colitis, Crohn's disease, hepatitis, pancreatitis, thyroid disease, and diabetes. Any information on specific diagnoses was checked against hospital records.

\subsection{Statistical Methods}

Univariate comparison of seropositive and seronegative responders was performed using the Chi-square test with Yates' correction, or Fisher's exact test for $2 \times 2$ contingency tables (both two-sided) in the computer program Epi Info (Centers for Disease Control, Atlanta, USA. To control for potential confounding effects of other risk factors, all variables with a $p$-value $<0.2$ in the univariate analysis were included in a multivariate generalized 
linear model using the procedure for binomial regression in STATA 8.0 (Stata Corporation, College Station, TX, USA). All results are expressed as relative risks (RR) with $95 \%$ confidence intervals $(\mathrm{CI})$ and two-tailed $p$ values.

\section{Results}

$433(=60.9 \%)$ (mean age 37.5 years, range $35-42$ years) of the 711 previous recruits answered the questionnaire. The response rate was higher among seropositives (79.7\%) than among seronegatives (58.9\%) in the 1987survey. Among the 433 responders, 51 had been seropositive for IgG or/and IgA in the 1987 survey, 380 had been seronegative, and 2 had ambiguous results (Table 1).

Regarding baseline characteristics, there was no statistically significant difference between the two groups that were seropositive viz. seronegative in the 1987 serosurvey, regarding level of education, annual income, or the following behavioral (lifestyle) factors: smoking, alcohol consumption, physical activity, and consumption of fruits, vegetables, dietary fibers, or red meat products (Table 2). Likewise, the seropositive enrollees were no more likely than the seronegative to have developed any of the following disorders since 1987: rheumatoid arthritis, ankylosing spondylitis, iridocyclitis, ulcerative colitis, Crohn's disease, hepatitis, pancreatitis, diabetes, or thyroid disease.

However, the seropositive enrollees were more likely to report chronic or recurrent diarrhea, painful or swollen joints, or muscle pains. Regarding frequencies of the particular complaints, totally $43 \%$ of seropositives complained of diarrhea, $27 \%$ of painful or swollen joints, and $27 \%$ of muscle pains. Corresponding frequencies among the 380 seronegatives were: diarrhea $16 \%$, painful or swollen joints $14 \%$, and muscle pains $12 \%$. Also was the concomitant presence of all three complaints signifi- cantly more frequent among the seropositives. Regarding the particular immunoglobulin subclasses, corresponding results were obtained, with the strongest correlations between IgA and clinical complaints (Table 3).

To assess whether previous Y. enterocolitica infection, as indicated by antibody status in 1987, was an independent risk factor for chronic diarrhea, painful or swollen joints, or muscle pains, we constructed separate multivariate models for each of these complaints. The results show that previous Y. enterocolitica infection was independently associated with increased risk; the RR estimates for $\mathrm{Y}$. enterocolitica as positive were not changed in multivariate analyses.

\section{Clinical Information Reported}

During the follow-up period, twenty-seven individuals had been admitted to hospital. There was no significant difference in admission frequency between the 1987groups of seropositive and seronegative recruits $(5 / 51$ $(9.8 \%) \approx 22 / 380(5.8 \%) ; p=0.34)$.

Three of the five seropositive individuals who had been hospitalized, had been admitted with complaints compatible with the previous Y. enterocolitica infection (diarrhea/joint complaints). The other two had undergone surgery for gangrenous appendicitis, viz. gastro-esophageal reflux disease.

Regarding the 22 seronegative recruits who had been admitted, two had developed ankylosing spondylitis, one rheumatoid arthritis, and in one patient acute knee joint arthritis was associated with iridocyclitis. Six patients experienced unspecific joint complaints.

Hence, the seropositive enrollees were no more likely than the seronegative to have developed any of the specific disorders asked for in the questionnaire (rheumatoid arthritis, ankylosing spondylitis, iridocyclitis, ulcerative colitis, Crohn's disease, hepatitis, pancreatitis, diabetes, or thyroid disease).

Table 1. Number of recruits in the 1987 serological survey and number of respondents in the 2004 follow up questionnaire study by Y.enterocolitica antibody activity in the 1987 survey.

\begin{tabular}{lccc}
\hline & IgG positive & IgG negative & Total \\
\hline 1987 serosurvey & 56 & 699 & 755 \\
2004 responders & & & 25 \\
IgA positive & 16 & 9 & 408 \\
IgA negative & 26 & 382 & 20 \\
IgM positive & 18 & $2^{\mathrm{a}}$ & 413 \\
IgM negative & 24 & 389 & 433 \\
2004 Follow up & 42 & 391 & \\
\hline
\end{tabular}

${ }^{\mathrm{a}}$ Excluded from statistical calculations because of single, ambiguous IgM titres. 
Table 2. Association between previous Yersinia antibody activity ${ }^{\mathrm{a}}$ and personal and demographic data, risk factors, and dietary parameters. Univariate analysis.

\begin{tabular}{|c|c|c|c|}
\hline Distribution of possible confounders & $\begin{array}{l}\text { Seropositive }{ }^{\mathrm{a}} \text { in } 1987 \\
\mathrm{~N}=51^{\mathrm{b}} \text { Number }(\%)\end{array}$ & $\begin{array}{l}\text { Seronegative in } 1987 \\
\mathrm{~N}=380^{\mathrm{b}} \text { Number }(\%)\end{array}$ & $\begin{array}{c}p \text {-value } \\
\text { (Fishers exact } 2 \text {-sided) }\end{array}$ \\
\hline - Annual income $>500,000$ (NOK) & $24(47)$ & $195 / 375(52)$ & 0.55 \\
\hline - Higher education (university, college) & $21 / 50(42)$ & $171 / 378(45)$ & 0.76 \\
\hline - Smoking & $18(35)$ & $127 / 376(34)$ & 0.88 \\
\hline - Alkohol consumption $\geq 1 /$ week & $16(31)$ & $140 / 379(37)$ & 0.54 \\
\hline \multicolumn{4}{|l|}{ - Physical activity } \\
\hline$<1$ time $/ \mathrm{w}$ & $22(43)$ & $190(50)$ & \\
\hline $1-3$ times $/ w$ & $18(35)$ & $151(40)$ & 0.077 \\
\hline$>3$ times $/ w$ & $11(22)$ & $39(10)$ & \\
\hline \multicolumn{4}{|l|}{ - Fruit consumption } \\
\hline$<1$ time $/ \mathrm{w}$ & $2(4)$ & $20(5)$ & \\
\hline $1-3$ times $/ w$ & $28(55)$ & $173(46)$ & 0.49 \\
\hline$>3$ times $/ w$ & $21(41)$ & $186(49)$ & \\
\hline \multicolumn{4}{|l|}{ - Fibre intake } \\
\hline$<1$ time $/$ w & $5(10)$ & $47(12)$ & \\
\hline $1-3$ times $/ \mathrm{w}$ & $14(27)$ & $119(31)$ & 0.73 \\
\hline$>3$ times $/ w$ & $32(63)$ & $214(56)$ & \\
\hline - Meat intake > 3 times/week & $9(18)$ & $43(11)$ & 0.14 \\
\hline
\end{tabular}

${ }^{\mathrm{a}} \mathrm{IgG}$ or IgA positive; ${ }^{\mathrm{b}}$ If not all have answered the question, the total number who answered is given in the table.

Table 3. Association between previous Yersinia antibody activity (classes IgG, IgA and IgM) and chronic disorders and complaints, univariate analysis.

\begin{tabular}{|c|c|c|c|c|c|}
\hline Chronic or recurrent complaints: & $\begin{array}{c}\text { Seropositive in } 1987 \\
\text { Number }(\%)\end{array}$ & $\begin{array}{l}\text { Seronegative }{ }^{\mathrm{a}} \text { in } \\
1987 \text { Number }(\%)\end{array}$ & Relative risk & $\begin{array}{c}95 \% \\
\text { Confidence interval }\end{array}$ & $p$-value \\
\hline IgG & 42 & 380 & & & \\
\hline - Diarrhea & $16(38)$ & $62(16)$ & 2.3 & $1.5-3.7$ & 0.0012 \\
\hline - Painful / swollen joints & $10(24)$ & $54(14)$ & 1.7 & $0.9-3.0$ & 0.156 \\
\hline - Muscle pains & $10(24)$ & $44(12)$ & 2.1 & $1.1-3.8$ & 0.045 \\
\hline - All three complaints & $4(10)$ & $12(3)$ & 3.02 & $1.0-8.9$ & 0.064 \\
\hline - Diarrhea or muscle or joint pain & $21(50)$ & $28(7)$ & 6.79 & $4.3-10.8$ & $<0.0001$ \\
\hline $\operatorname{IgA}$ & 25 & 380 & & & \\
\hline - Diarrhea & $12(48)$ & $62(16)$ & 2.9 & $1.8-4.7$ & $<0.0004$ \\
\hline - Painful / swollen joints & $8(32)$ & $54(14)$ & 2.3 & $1.2-4.2$ & $0.038)$ \\
\hline - Muscle pains & $10(40)$ & $44(12)$ & 3.5 & $2.0-6.0$ & $<0.001$ \\
\hline - All three complaints & $5(20)$ & $12(3)$ & 6.33 & $2.4-16.6$ & 0.0022 \\
\hline - Diarrhea or muscle or joint pain & $15(60)$ & $28(7)$ & 8.14 & $5.0-13.2$ & $<0.0001$ \\
\hline $\operatorname{IgM}$ & 18 & 380 & & & \\
\hline - Diarrhea & $6(33)$ & $62(16)$ & 2.0 & $1.0-4.0$ & 0.10 \\
\hline - Painful / swollen joints & $5(28)$ & $54(14)$ & 2.0 & $0.9-4.3$ & 0.16 \\
\hline - Muscle pains & $6(33)$ & $44(12)$ & 2.9 & $1.4-5.9$ & 0.017 \\
\hline - All three complaints & $2(11)$ & $12(3)$ & 3.52 & $0.85-14.6$ & 0.13 \\
\hline - Diarrhea or muscle or joint pain & $10(55)$ & $28(7)$ & 7.54 & $4.4-13.0$ & $<0.0001$ \\
\hline Any antibody & 51 & 380 & & & \\
\hline - Diarrhea & $22(43)$ & $62(16)$ & 2.64 & $1.8-3.9$ & $<0.0001$ \\
\hline - Painful / swollen joints & $14(27)$ & $54(14)$ & 1.93 & $1.2-3.2$ & 0.025 \\
\hline - Muscle pains & $14(27)$ & $44(12)$ & 2.4 & $1.4-4.0$ & 0.004 \\
\hline - All three complaints & $7(14)$ & $12(3)$ & 4.35 & $1.8-10.5$ & 0.004 \\
\hline - Diarrhea or muscle or joint pain & $28(55)$ & $28(7)$ & 7.45 & $4.8-11.5$ & $<0.0001$ \\
\hline
\end{tabular}

${ }^{a}$ Seronegative for IgG, IgA and IgM. 


\section{Discussion}

433 of the 711 previous recruits answered the questionnaire, with the higher response rate among seropositives. The difference in response rate may to a certain extentbe attributable to higher interest among seropositives, occasioned by higher frequencies of complaints. During the period of observation, several recruits with complaints (or their doctors) had inquired about their antibody state, or asked for clinical advice.

In 1987, none of the recruits (mean age 20 years) suffered from acute $\mathrm{Y}$. enterocolitica infection, or were aware of previous infection. A relatively stable incidence among children and youngsters would indicate that the seropositive recruits might have acquired their infection about ten years prior to enrolment. At enrolment, their clinical and serological status might mirror only a 10-year course, explaining why recurrent diarrhea or joint complaints were not associated with antibody activity [19]. This suggestion is in concert with the specific time information of 10 - 13 years obtained in case of the four seroposive recruits operated upon because of mesenteric lymphadenitis. In these cases, surgery most certainly coincides with the acute infection, as mesenteric lymphadenitis is its most commonly encountered abdominal manifestation in childhood [20]. At enrolment, therefore, the recruits' antibody activity might have persisted for 11 13 years. Corresponding observations on persistence of antibodies for $>10$ years have previously been made in long-time follow-up studies [5,13].

At follow-up 2004, may be on average 27 years after catching their infection, the 1987-seropositive recruits were significantly more likely to report chronic or recurrent diarrhea, painful or swollen joints, or muscle pains, than were their 1987-seronegative counterparts. The presence of all three complaints was also more frequent among the group of seropositives. Regarding frequencies of particular complaints, $43 \%$ of seropositives complained of diarrhea, $27 \%$ of painful or swollen joints, and $27 \%$ of muscle pains. These frequencies correspond well with those observed in our 10-year follow-up study on hospitalized patients (37\% of questionnaire repliers suffered recurrent diarrhea, 44\% joint complaints) (5); further are the observations in accordance with several previous reports on hospitalized patients [4,11,21,22]. Patients with long-time observation may present the higher frequencies of particular complaints; supporting the concept of development over several years [20].

In contrast to the observations in a Finnish follow-up study on conscripts who experienced acute Y. enterocolitica infection in their garrison [13], none of our seropositive recruits developed chronic rheumatic disease attributable to the infection. However, individuals prone to a serious course might have developed their chronic condition before attending at the military medical board, and in consequence been excluded from military service.

During the 17-year long follow-up period, some 1987seronegative recruits may have acquired Y. enterocolitica infection, and thereby possibly contributed to particular complaints reported by "seronegative" recruits at followup. We must leave this question open, as the follow-up examination did not include estimation of antibody activity.

In the present study, the immunoglobulin subclass IgA showed the stronger association with chronic clinical complaints. A corresponding observation was made already in 1980, in a follow-up study on patients with, or without, reactive arthritis [23]. In 1985, it was stated that determination of $\operatorname{IgA}$ antibodies (which have a very short half-life) was of prognostic value in the evaluation of the infection's chronicity [24]; and evidence for year-long persistence of $\mathrm{Y}$. enterocolitica in the human body was demonstrated. The chronically elevated IgA production is recognized as the result of chronic stimulation of the gutassociated lymphoid tissue by virulent persistent Yersinia antigen [14]. A few contributions indicate that long-time antibiotic treatment, if started in time and lasting long enough, may eliminate Y. enterocolitica from the gutassociated lymphoid tissue, with subsequent disappearance of IgA antibodies and relief of clinical disease [25].

\section{Conclusions}

The present study shows that previous uncomplicated Y. enterocolitica infection is a risk factor regarding development of chronic complaints as diarrhea, painful or swollen joints, and muscle pain. Especially, the predictive value of IgA should be noticed.

\section{REFERENCES}

[1] M. G. Carlsson, H. Ryd and N. H. Sternby, "A Case of Human Infection with Pasteurellapseudotuberculosis X," Acta Pathologica Microbiologica Scandinavica, Vol. 62, 1964, pp. 128-132.

[2] B. Nilehn and B. Sjöström, "Studies on Yersinia Enterocolitica. Occurence in Various Groups of Acute Abdominal Disease," Acta Pathologica Microbiologica Scandinavica, Vol. 71, No. 4, 1967, pp. 612-628. doi:10.1111/j.1699-0463.1967.tb05182.x

[3] B. Arvastson, K. Damgaard and S. Winblad, "Clinical Symptoms of Infection with Yersinia Enterocolitica," Scandinavian Journal of Infectious Diseases, Vol. 3, No. 1, 1971, pp. 37-40.

[4] P. Ahvonen, "Human Yersiniosis in Finland, II. Clinical Features," Annals of Clinical Research, Vol. 4, No. 1, 1972, 
pp. $39-48$.

[5] A. Saebo and J. Lassen, "A Survey of Acute and Chronic Disease Associated with Yersinia Enterocolitica Infection. A Norwegian 10-year Follow-up Study on 458 Hospitalized Patients," Scandinavian Journal of Infectious Diseases, Vol. 23, No. 5, 1991, pp. 517-527. doi:10.3109/00365549109105172

[6] K. Aho, P. Ahvonen, A. Lassus, K. Sievers and A. Tiilikainen, "HL-A27 in Reactive Arthritis. A Study of Yersinia Arthritis and Reiter's Disease," Arthritis \& Rheumatism, Vol. 17, No. 5, 1974, pp. 521-526. doi:10.1002/art.1780170505

[7] M. Leirisalo, G. Skylv, M. Kousa, L. M. Voipo-Pulkki , H. Souranta, M. Nissila, et al., "Follow-up Study on Patients with Reiter's Disease and Reactive Arthritis, with Special Reference to HLA-B27," Arthritis \& Rheumatism, Vol. 25, No. 3, 1982, pp. 249-259. doi:10.1002/art.1780250302

[8] M. Friedberg, T. Denneberg, C. Brun, J. H. Larsen, S. Larsen, "Glomerulonephritis in Infection with Yersinia Enterocolitica O-serotype 3. II. The Incidence and Immunological Features of Yersinia Infection in a Consecutive Glomerulonephritis Population," Acta Medica Scandinavica, Vol. 209, No. 1-6, 1981, pp. 103-110. doi:10.1111/j.0954-6820.1981.tb11560.x

[9] K. A. Sotaniemi, "Neurologic Complications Associated with Yersiniosis," Neurology, Vol. 33, No. 1, 1983, pp. 95-97.

[10] A. Saebo, "Liver Affection Associated with Yersinia Enterocolitica Infection," Acta Chirurgica Scandinavica, Vol. 143, No. 7-8, 1977, pp. 445-450.

[11] A. Saebo, E. Vik, O. J. Lange and L. Matuszkiewicz, "Inflammatory Bowel Disease Associated with Yersinia Enterocolitica O:3 Infection," European Journal of Internal Medicine, Vol. 16, No. 3, 2005, pp. 176-182. doi:10.1016/j.ejim.2004.11.008

[12] M. Leirisalo-Repo and H. Souranta, "Ten-Year Follow-up Study of Patients with Yersinia Arthritis," Arthritis \& Rheumatism, Vol. 31, No. 4, 1988, pp. 533-537. doi:10.1002/art.1780310410

[13] H. Lindholm and R. Visakorpi, "Late Complications after a Yersinia Enterocolitica Epidemic: A Follow up Study". Annals of the Rheumatic Diseases, Vol. 50, No. 10, 1991, pp. 694-696. doi:10.1136/ard.50.10.694

[14] J. A. A. Hoogkamp-Korstanje, J. de Koning and J. Heesemann, "Persistence of Yersinia Enterocolitica in Man," Infection, Vol. 16, No. 2, 1988, pp. 81-85. doi:10.1007/BF01644307

[15] L. Vold and K. Nygard, "Yersiniosis in Norway 2002," MSIS-rapport, (In Norwegian), Communicable Disease Report, Norwegian Institute of Public Health, Oslo, Vol. 31,2003 , p. 40.
[16] F. Kallinowski, A. Wassmer, M. A. Hoffmann, D. Harmsen, J. Heesemann, H. Karch, et al., "Prevalence of Enteropatogenic Bacteria in Surgically Treated Chronic Inflammatory Bowel Disease," Heptogastroenterology, Vol. 45, No. 23, 1998, pp. 1552-1558.

[17] J. A. Hoogkamp-Korstanje, J. de Koning, J. Heesemann, J. J. Festen, P. M. Houtman and P. L. van Oyen, "Influence of Antibiotics on IgA and IgG Response and Persistence of Yersinia Enterocolitica in Patients with YersiniaAssociated Spondylarthropathy," Infection, Vol. 20, No. 2, 1992, pp. 53-57. doi:10.1007/BF01711062

[18] B. E. Wenzel, J. Heesemann, A. Heufelder, T. F. Franke S. Grammerstorf, R. Stemerowicz, et al., "Enteropathogenic Yersinia Enterocolitica and Organ-Specific Autoimmune Disease in Man," Contrib Microbiol Immunol, Vol. 12, 1991, pp. 80-88.

[19] A. Saebo, G. Kapperud, J. Lassen and J. O. Waage "Prevalence of Antibodies to Yersinia Enterocolitica O:3 among Norwegian Military Recruits: Association with Risk Factors and Clinical Manifestations," European Journal of Epidemiology, Vol. 10, No. 6, 1994, pp. 749-755. doi:10.1007/BF01719293

[20] A. Saebo and J. Lassen, "Acute and chronic Gastrointestinal Manifestations Associated with Yersinia Infection. A Norwegian 10-year Follow-up Study on 458 Hospitalized Patients," Annals of Surgery, Vol. 215, No. 3, 1992, pp. 250-255. doi:10.1097/00000658-199203000-00009

[21] G. Vantrappen, E. Ponette, K.Geboes and P. Bertrand, "Yersinia Enteritis and Enterocolitis: Gastroenterological Aspects," Gastroenterology, Vol. 72, No. 2, 1977, pp. 220-227.

[22] A. Saebo, "The Yersinia Enterocolitica Infection in Acute Abdominal Surgery: A Clinical Study with a 5 Year Follow-up Period," Annals of Surgery, Vol. 198, No. 6, 1983, pp. 760-765. doi:10.1097/00000658-198312000-00015

[23] K. Granfors, M. Viljanen, A. Tiilikainen and A. Toivanen "Persistence of IgM, IgG, and IgA antibodies to Yersinia in Yersinia arthritis," Journal of Infectious Diseases, Vol. 141, No. 4, 1980, pp. 424-249. doi:10.1093/infdis/141.4.424

[24] J. H. Larsen, S. H. Hartzen and M. Parm, "The Determination of Specific IgA-Antibodies to Yersinia Enterocolitica and their Role in Enteric Infections and their Complications," Acta Pathologica Microbiologica Scandinavica Series B: Microbiology, Vol. 93, No. 1-6, 1985, pp. 331-339.

[25] J. A. A. Hoogkamp-Korstanje, H. Moesker, G. A. W. Bruyn, "Ciprofloxacin v Placebo for Treatment of Yersinia Enterocolitica Triggered Reactive Arthritis," Annals of the Rheumatic Diseases, Vol. 59, No. 11, 2000, pp. 914-917. doi:10.1136/ard.59.11.914 